Change in Prescribing forSecondary Prevention of Stroke and Coronary Heart Disease in Finnish Nursing Homes and Assisted Living Facilities

Jokanovic, Natali

2019-06

Jokanovic , N , Kautiainen , H , Bell , J S , Tan , E C K \& Pitkälä , K H 2019 , ' Change in Prescribing forSecondary Prevention of Stroke and Coronary Heart Disease in Finnish Nursing Homes and Assisted Living Facilities ' , Drugs \& Aging , vol. 36 , no. 6 , pp. 571-579 . https://doi.org/10.1007/s40266-019-00656-x

http://hdl.handle.net/10138/316131

https://doi.org/10.1007/s40266-019-00656-x

unspecified

publishedVersion

Downloaded from Helda, University of Helsinki institutional repository.

This is an electronic reprint of the original article.

This reprint may differ from the original in pagination and typographic detail.

Please cite the original version. 


\title{
Change in Prescribing for Secondary Prevention of Stroke and Coronary Heart Disease in Finnish Nursing Homes and Assisted Living Facilities
}

\author{
Natali Jokanovic ${ }^{1,2} \cdot$ Hannu Kautiainen ${ }^{3} \cdot$ J. Simon Bell ${ }^{1} \cdot$ Edwin C. K. $\operatorname{Tan}^{1,4,5} \cdot$ Kaisu H. Pitkälä ${ }^{3}$
}

Published online: 5 April 2019

(c) Springer Nature Switzerland AG 2019

\begin{abstract}
Background One quarter of residents in long-term care facilities (LTCFs) have a diagnosis of CHD or stroke and over half use at least one preventative cardiovascular medication. There have been no studies that have investigated the longitudinal change in secondary preventative cardiovascular medication use in residents in LTCFs over time.

Objective The aim of this study was to investigate the change in cardiovascular medication use among residents with coronary heart disease (CHD) and prior stroke in nursing homes (NHs) and assisted living facilities (ALFs) in Finland over time, and whether this change differs according to dementia status.

Methods Three comparable cross-sectional audits of cardiovascular medication use among residents aged 65 years and over with CHD or prior stroke in NHs in 2003 and 2011 and ALFs in 2007 and 2011 were compared. Logistic regression analyses adjusted for gender, age, mobility, cancer and length of stay were performed to examine the effect of study year, dementia and their interaction on medication use.

Results Cardiovascular medication use among residents with CHD (NHs: 89\% vs 70\%; ALFs: 89\% vs 84\%) and antithrombotic medication use among residents with stroke (NHs: 72\% vs 63\%; ALFs: 78\% vs 69\%) declined between 2003 and 2011 in NHs and 2007 and 2011 in ALFs. Decline in the use of diuretics, nitrates and digoxin were found in both groups and settings. Cardiovascular medication use among residents with CHD and dementia declined in NHs (88\% [95\% CI 85-91] in 2003 vs 70\% [95\% CI 64-75] in 2011) whereas there was no change among people without dementia. There was no change in cardiovascular medication use among residents with CHD in ALFs with or without dementia over time. Antithrombotic use was lower in residents with dementia compared with residents without dementia in NHs $(p<0.001)$ and ALFs $(p=0.026)$; however, the interaction between dementia diagnosis and time was non-significant.

Conclusions The decline in cardiovascular medication use in residents with CHD and dementia suggests Finnish physicians are adopting a more conservative approach to the management of cardiovascular disease in the NH population.
\end{abstract}

\section{Introduction}

Coronary heart disease (CHD) and stroke contribute to $45 \%$ and $17 \%$ of all deaths attributable to cardiovascular disease in the United States (US) [1]. One quarter of residents in long-term care facilities (LTCFs) have a diagnosis of CHD or stroke and over half use at least one preventative cardiovascular medication [2-4]. Cardiovascular disease is a

Electronic supplementary material The online version of this article (https://doi.org/10.1007/s40266-019-00656-x) contains supplementary material, which is available to authorized users.

Natali Jokanovic

Natali.Jokanovic@monash.edu

Extended author information available on the last page of the article

\section{Key Points}

Significant decline in the use of cardiovascular medications among residents with coronary heart disease (CHD) and antithrombotic medications among residents with a prior history of stroke in Finnish nursing homes (NHs) and assisted living facilities was observed over an 8-year period.

Decline in the use of cardiovascular medications among residents with CHD in NHs may be explained by the increasing proportion of residents with dementia.

Findings suggest conservative pharmacological management of cardiovascular disease among Finnish NH residents with dementia and CHD. 
significant contributor to disability, functional decline, complex care requirements and healthcare costs in LTCFs $[5,6]$.

Between 13 and $74 \%$ of residents in LTCFs take nine or more regular medications [7]. Half of residents are prescribed antithrombotics and up to $80 \%$ are prescribed at least one cardiovascular agent [7-9]. Compared with older people in the community, residents of LTCFs are less likely to be prescribed medications for stroke (aspirin and other anticoagulants) and for heart disease [ $\beta$-blockers, angiotensinconverting enzyme inhibitors (ACEIs) and aspirin] [10, 11]. At least $50 \%$ of residents in US and European LTCFs have diagnosed dementia. Clinicians may adopt a more conservative approach to prescribing for residents with dementia who they perceive are at a high risk of adverse drug events (ADEs) $[12,13]$. A palliative approach with focus on optimizing quality of life in LTCF residents with dementia who have limited life expectancy has been advocated [14]. This trend is evident in US outpatient clinics where antihypertensive prescribing for people aged 65 years and over with dementia has remained stable despite an increase in the number of people with hypertension [15].

Prescribing recommendations in clinical practice guidelines for the secondary prevention of CHD and stroke are not specific to patients' age and setting [16, 17]. The 2011 American Heart Association (AHA) and American College of Cardiology Foundation (ACCF) guidelines and the 2013 European Society of Cardiology (ESC) guidelines recommend the use of ACEIs or angiotensin II receptor blockers (ARBs), $\beta$-blockers, statins and antiplatelet agents for the secondary prevention of CHD in all populations $[16,18]$. The 2014 AHA and American Stroke Association (ASA) guidelines recommend the use of antithrombotic agents in addition to pharmacotherapy to manage risk factors such as atrial fibrillation and hypertension [17]. Although the number of clinical trials including the general older population as participants are increasing, the evidence base for managing CHD and stroke among residents in LTCFs is limited. Recent trials investigating antihypertensive therapy specifically in older people [19] include the Hypertension in the Very Elderly Trial (HYVET) and Systolic Blood Pressure Intervention Trial (SPRINT) in community-dwelling older people aged over 80 and 75 years, respectively [20, 21], and the Predictive Values of Blood Pressure and Arterial Stiffness in Institutionalized Very Aged Population (PARTAGE) study in LTCF residents aged over 80 years [22]. Although evidence is limited and conflicting over the benefit and harm of antihypertensive therapy in the frail older population [19], their use is recommended after careful consideration of comorbidity and potential ADEs [23]. Prescribing decisions in LTCFs continue to rely on individualized consideration of multimorbidity, polypharmacy, frailty, life expectancy and the increased risk of ADEs [24, 25].
Recent studies in European and US LTCFs have explored secondary preventative cardiovascular medication use. A US study in LTCFs identified $37 \%$ of residents were not prescribed cardiovascular medications following an acute myocardial infarction within 30 days of readmission to the LTCF [26]. The Services and Health for Elderly in LongTerm care (SHELTER) study identified $77.7 \%$ of residents with CHD received a cardiovascular medication (ACEI or ARB, $\beta$-blocker, antiplatelet or statin) [3]. Additionally, between 24.6 and $60.5 \%$ of residents with CHD in Polish LTCFs were prescribed either aspirin, $\beta$-blockers, ACEI or statins [27]. No studies have investigated the longitudinal change in secondary preventative cardiovascular medication use in residents in LTCFs over time. This knowledge is needed to determine whether changing attitudes to prescribing in older people with limited life expectancy has influenced prescribing $[14,28]$.

The objective of this study was to investigate the change in cardiovascular medication use among residents with stroke and CHD in nursing homes (NHs) and assisted living facilities (ALFs) in Finland between 2003 and 2011, and whether this change differs according to dementia status.

\section{Methods}

\subsection{Study Design, Setting and Source}

Data from three previous cross-sectional studies exploring nutrition and medication use in NHs and ALFs in Helsinki, Finland were combined. The methodology of these studies has been described previously [29-32]. In brief, data were obtained from all residents aged 65 years and over who resided permanently in NHs in Helsinki in $2003(n=1987)$ and $2011(n=1576)$, and ALFs in $2007(n=1377)$ and 2011 $(n=1586)$. Baseline characteristics of the combined dataset are reported elsewhere [31]. Data for residents with CHD and stroke were extracted from the combined dataset. A total of 2012 residents with CHD and 1755 residents with a prior history of stroke were included in the current study.

Although the terms 'LTCF', 'NH' and 'ALF' are used interchangeably in the literature to define a facility providing 24-h care to individuals who can no longer reside at home, differences in resident and facility characteristics exist internationally [33]. In this study, NHs are facilities that provide 24-h care to individuals who have complex care needs that can no longer be met in the community [33]. ALFs provide similar 24-h care by a registered nurse but in a more homelike environment, including apartments and group homes [31]. 


\subsection{Study Sample and Data Collection}

Trained nurses collected data using the same data collection tools and methodology across all three studies. Data collected included resident demographics, medication information and medical diagnoses obtained from the residents' medication charts and medical records. Medication use was measured as a point prevalence on the day of data collection.

\subsection{Measures and Definitions}

All medications were coded using the Anatomical Therapeutic Chemical (ATC) classification [34]. All regular prescription and non-prescription medications were recorded. Medications were considered to be regular if there was a documented regular sequence of administration. Cardiovascular agents included $\beta$-blockers (C07), agents acting on the renin-angiotensin system (C09), calcium channel blockers (C08), diuretics (C03), organic nitrates (C01DA), lipid modifying agents (C10) and digoxin (C01AA05). Antithrombotic agents included warfarin (B01AA03), dipyridamole (B01AC07), dipyridamole in combination with aspirin (B01 AC30) and low-dose aspirin (B01AC06). For combination products, each active ingredient was counted separately. Polypharmacy was defined as the use of nine or more regular medications. A resident was considered to have dementia if the diagnosis was listed in the medical record or the resident used an anti-dementia medication (N06A). The Charlson comorbidity index (CCI), a weighted measure incorporating up to 19 comorbidities, was calculated for each resident [35]. Nutritional status was calculated using the Mini Nutritional Assessment (MNA) [36]. A score of $<17$ was regarded as an indication of risk of malnutrition.

\subsection{Statistical Analysis}

Analyses were performed separately for residents who had a reported history of CHD and prior stroke in NHs between the years 2003 and 2011 and in ALFs between 2007 and 2011. Resident demographics, clinical characteristics and cardiovascular medication use across time periods were compared using Chi square tests for categorical variables, Student's t-tests for continuous variables and Mann-Whitney $U$ tests for non-parametric continuous variables.

Logistic regression analyses were performed to examine the effect of study year, dementia and their interaction (study year $\times$ dementia) on the use of any cardiovascular or antithrombotic agent for residents with CHD or stroke in $\mathrm{NHs}$ and ALFs, respectively. The analyses were adjusted for gender, age, mobility, cancer and length of stay in the facility, which were deemed to be clinically relevant [9]. Adjusted percentages of cardiovascular and antithrombotic medication use were estimated from logistic regression analyses. All statistical analyses were performed using Stata 14.1 (StataCorp, College Station, TX, USA).

\section{Results}

The prevalence of CHD decreased over time in both NHs (35.5\% in 2003 to $24.5 \%$ in 2011) and ALFs (34.4\% in 2007 to $28.2 \%$ in 2011) whereas the prevalence of prior history of stroke remained constant in NHs (27.2\% in 2003 to $28.6 \%$ in 2011) and in ALFs (25.8\% in 2007 to 25.7\% in 2011).

\subsection{Residents with Coronary Heart Disease}

Residents with CHD were older, had greater disability, were more likely to be at risk of malnourishment, have dementia and greater comorbidity in both NHs and ALFs in 2011 compared with respective baselines (Table 1, Supplementary Figure S1, see electronic supplementary material [ESM]). The prevalence of polypharmacy decreased in NHs (54.4\% vs $41.2 \% ; p<0.001)$ but increased in ALFs $(55.0 \%$ vs $62.2 \% ; p=0.026)$.

The use of any cardiovascular agent significantly declined in both settings among residents with CHD. Significant decline in the use of diuretics, nitrates and digoxin were identified in NHs in 2011 compared with baseline. Similar trends were seen in ALFs concerning nitrates and digoxin. The use of any antithrombotic agent and use of low-dose aspirin significantly declined in NHs only.

\subsection{Residents with a Prior History of Stroke}

Residents with a history of stroke were more likely to have greater disability and comorbidity in NHs and ALFs in 2011 compared with baseline (Table 2, Supplementary Figure S2, see ESM). Polypharmacy significantly decreased in NHs ( $47.1 \%$ vs $37.5 \%: p=0.0022)$.

The use of any antithrombotic agent significantly decreased in both NHs and ALFs. Specifically, the use of low-dose aspirin and dipyridamole significantly declined in both settings. The use of warfarin and statins increased, although only significantly in NHs. Reductions in the overall use of any cardiovascular agent were observed in both settings among residents with a history of stroke.

\subsection{Interaction of Dementia and Study Year on Cardiovascular and Antithrombotic Use}

Adjusted percentages of cardiovascular and antithrombotic medication use in residents with a history of CHD and stroke according to dementia status, respectively, are shown in Fig. 1. Findings from the logistic regression analyses are reported in Supplementary Tables S1 and S2 (see ESM). Of 
Table 1 Characteristics of residents in nursing homes and assisted living facilities with coronary heart disease

\begin{tabular}{|c|c|c|c|c|c|c|}
\hline Characteristics & $\begin{array}{l}\text { Nursing } \\
\text { homes in } \\
2003 \\
n=706\end{array}$ & $\begin{array}{l}\text { Nursing } \\
\text { homes in } \\
2011 \\
n=386\end{array}$ & $p$ value & $\begin{array}{l}\text { Assisted living } \\
\text { facilities in } 2007 \\
n=473\end{array}$ & $\begin{array}{l}\text { Assisted living } \\
\text { facilities in } 2011 \\
n=447\end{array}$ & $p$ value \\
\hline \multicolumn{7}{|l|}{ Demographic and clinical characteristics } \\
\hline Females, $n(\%)$ & $569(80.6)$ & $294(76.2)$ & 0.086 & $369(78.0)$ & $351(78.5)$ & 0.85 \\
\hline Education $<8$ years, $n(\%)$ & $368(59.9)$ & $159(50.0)$ & 0.004 & $248(56.8)$ & $230(56.2)$ & 0.88 \\
\hline Mean age (SD) & $85.3(7.0)$ & $86.7(7.0)$ & $<0.001$ & $84.4(6.7)$ & $85.9(6.7)$ & 0.002 \\
\hline Not able to move independently, $n(\%)$ & 209 (29.7) & $217(56.2)$ & $<0.001$ & $72(15.2)$ & $128(28.6)$ & $<0.001$ \\
\hline Widowed, $n(\%)$ & $414(59.2)$ & $214(56.8)$ & 0.43 & $294(62.8)$ & $285(65.4)$ & 0.43 \\
\hline \multicolumn{7}{|l|}{ MNA, $n(\%)$} \\
\hline Malnourished (<17 points) & $208(29.5)$ & $119(30.8)$ & $<0.001$ & $53(11.2)$ & $89(20.0)$ & 0.001 \\
\hline Charlson comorbidity index (SD) & $2.9(1.2)$ & $2.7(1.6)$ & $<0.001$ & $2.5(1.5)$ & $2.7(1.7)$ & 0.30 \\
\hline Prior stroke, $n(\%)$ & $239(36.8)$ & $121(31.3)$ & 0.076 & $147(31.1)$ & $126(28.2)$ & 0.33 \\
\hline Dementia, $n(\%)$ & $464(65.7)$ & $305(79.0)$ & $<0.001$ & $267(56.4)$ & $314(70.2)$ & $<0.001$ \\
\hline \multicolumn{7}{|l|}{ Medication use } \\
\hline Mean no. of medications (SD) & $9.2(3.4)$ & $8.2(3.4)$ & $<0.001$ & $8.9(3.4)$ & $9.8(3.7)$ & $<0.001$ \\
\hline Polypharmacy ( $\geq 9$ meds), $n(\%)$ & $384(54.4)$ & $159(41.2)$ & $<0.001$ & $260(55.0)$ & $278(62.2)$ & 0.026 \\
\hline Any cardiovascular agent, $n(\%)$ & $629(89.1)$ & $270(69.9)$ & $<0.001$ & $421(89.0)$ & $376(84.1)$ & 0.029 \\
\hline$\beta$-blocker, $n(\%)$ & $292(41.1)$ & $163(42.2)$ & 0.78 & $250(52.9)$ & $207(46.3)$ & 0.047 \\
\hline ACEI/ARB, $n(\%)$ & $153(21.7)$ & $103(26.7)$ & 0.062 & $180(38.1)$ & $165(36.9)$ & 0.72 \\
\hline Ca channel blocker, $n(\%)$ & $74(10.5)$ & $31(8.0)$ & 0.19 & $101(21.4)$ & $60(13.4)$ & 0.002 \\
\hline Diuretics, $n(\%)$ & $419(59.3)$ & $139(36.0)$ & $<0.001$ & $254(53.7)$ & $217(48.5)$ & 0.12 \\
\hline Nitrates, $n(\%)$ & $308(43.6)$ & $34(8.8)$ & $<0.001$ & $153(32.3)$ & $78(17.4)$ & $<0.001$ \\
\hline Statins, $n(\%)$ & $20(2.8)$ & $29(7.5)$ & $<0.001$ & $101(21.4)$ & $120(26.8)$ & 0.051 \\
\hline Digoxin, $n(\%)$ & $131(18.6)$ & $14(3.6)$ & $<0.001$ & $68(14.4)$ & $25(5.6)$ & $<0.001$ \\
\hline Mean no. of cardiovascular medications (SD) & $2.0(1.2)$ & $1.3(1.2)$ & $<0.001$ & $2.3(1.5)$ & $2.0(1.3)$ & $<0.001$ \\
\hline Any antithrombotic agents, $n(\%)$ & $490(69.4)$ & $235(60.9)$ & 0.004 & $361(76.3)$ & $327(73.2)$ & 0.27 \\
\hline Low-dose aspirin, $n(\%)$ & $389(55.1)$ & $153(39.6)$ & $<0.001$ & $240(50.7)$ & $203(45.4)$ & 0.11 \\
\hline
\end{tabular}

$A C E I$ angiotensin-converting enzyme inhibitor, $A R B$ angiotensin II receptor blocker, $M N A$ mini nutritional assessment, $S D$ standard deviation

NH residents with CHD, the use of any cardiovascular agent decreased over time in those with dementia from $88 \%(95 \%$ CI 85-91) in 2003 to $70 \%$ (95\% CI 64-75) in 2011, whereas no difference was found among residents without dementia (91\% [95\% CI 88-95] in 2003 vs $89 \%$ [95\% CI 83-95] in 2011); $p=0.0017$ for study year; $p<0.001$ for dementia diagnosis; $p=0.050$ for their interaction. Although the difference between cardiovascular medication use in residents with and without dementia in ALFs was significant, there was no difference in their use over time in residents with dementia ( $87 \%$ [95\% CI 83-91] in 2007 vs $84 \%$ [95\% CI $80-88$ ] in 2011) or without dementia (91\% [95\% CI 87-95] in 2007 vs 91\% [95\% CI 86-96] in 2011); $p=0.66$ for study year; $p=0.026$ for dementia diagnosis; $p=0.47$ for their interaction).

Of NH and ALF residents with a prior history of stroke, the difference in the use of any antithrombotic agent in residents with and without dementia was significant, however their change in use over time was not. Of NH residents with a prior history of stroke, the use of any antithrombotic agent decreased over time in residents with dementia from $68 \%$ (95\% CI 63-73) in 2003 to $61 \%$ (95\% CI 55-66) in 2011 and without dementia from $81 \%$ (95\% CI 75-87) in 2003 to $72 \%$ (95\% CI 65-80) in 2011 ( $p=0.013$ for study year,; $p<0.001$ for dementia diagnosis; $p=0.61$ for their interaction). In ALFs the respective figures were 74\% (95\% CI 67-80) in 2007 versus 67\% (95\% CI 61-73) in 2011 among residents with dementia and 82\% (95\% CI 76-88) in 2007 versus $75 \%$ (95\% CI 68-82) in 2011 for residents without dementia ( $p=0.036$ for study year; $p=0.015$ for dementia diagnosis; $p=0.81$ for their interaction).

\section{Discussion}

Trends over an 8-year period in Helsinki NHs and ALFs reveal significant changes in the use of cardiovascular and antithrombotic agents in residents with a history of CHD 
Table 2 Characteristics of residents in nursing homes and assisted living facilities with a prior history of stroke

\begin{tabular}{|c|c|c|c|c|c|c|}
\hline Characteristics & $\begin{array}{l}\text { Nursing } \\
\text { homes in } \\
2003 \\
n=541\end{array}$ & $\begin{array}{l}\text { Nursing } \\
\text { homes in } \\
2011 \\
n=451\end{array}$ & $p$ value & $\begin{array}{l}\text { Assisted living } \\
\text { facilities in } 2007 \\
n=355\end{array}$ & $\begin{array}{l}\text { Assisted living } \\
\text { facilities in } 2011 \\
n=408\end{array}$ & $p$ value \\
\hline \multicolumn{7}{|l|}{ Demographic and clinical characteristics } \\
\hline Females, $n(\%)$ & $419(77.4)$ & $327(72.5)$ & 0.073 & $255(71.8)$ & $292(71.6)$ & 0.94 \\
\hline Education $<8$ years, $n(\%)$ & $296(61.2)$ & $181(50.6)$ & 0.002 & $192(58.7)$ & $183(48.9)$ & 0.01 \\
\hline Mean age (SD) & $82.9(7.4)$ & $83.2(7.8)$ & 0.45 & $82.0(7.7)$ & $82.8(7.8)$ & 0.28 \\
\hline Not able to move independently, $n(\%)$ & $232(43.0)$ & $312(69.2)$ & $<0.001$ & $89(25.1)$ & $177(43.4)$ & $<0.001$ \\
\hline Widowed, $n(\%)$ & $270(50.4)$ & $223(49.8)$ & 0.85 & $197(57.1)$ & $221(55.3)$ & 0.61 \\
\hline \multicolumn{7}{|l|}{ MNA, $n(\%)$} \\
\hline Malnourished (<17 points) & $168(31.1)$ & $143(31.7)$ & 0.26 & $42(11.8)$ & $104(25.6)$ & $<0.001$ \\
\hline Charlson comorbidity index (SD) & $2.8(1.2)$ & $3.9(1.3)$ & $<0.001$ & $3.6(1.2)$ & $3.9(1.4)$ & 0.002 \\
\hline Coronary heart disease, $n(\%)$ & $239(44.2)$ & $121(26.8)$ & $<0.001$ & $147(41.4)$ & $126(30.9)$ & 0.003 \\
\hline Dementia, $n(\%)$ & $351(64.9)$ & $304(67.4)$ & 0.40 & $196(55.2)$ & $247(60.5)$ & 0.14 \\
\hline \multicolumn{7}{|l|}{ Medication use } \\
\hline Mean no. of medications (SD) & $8.5(3.6)$ & $7.7(3.4)$ & 0.001 & $8.6(3.6)$ & $9.1(3.8)$ & 0.042 \\
\hline Polypharmacy ( $\geq 9$ meds), $n(\%)$ & $255(47.1)$ & $169(37.5)$ & 0.002 & $182(51.3)$ & $222(54.4)$ & 0.39 \\
\hline Any cardiovascular agents, $n(\%)$ & $399(73.8)$ & $284(63.0)$ & 0.37 & $284(80.0)$ & $282(69.1)$ & $<0.001$ \\
\hline$\beta$-blocker, $n(\%)$ & $178(32.9)$ & $155(34.4)$ & 0.63 & $148(41.7)$ & $145(35.5)$ & 0.081 \\
\hline ACEI/ARB, $n(\%)$ & $97(17.9)$ & $109(24.2)$ & 0.016 & $107(30.1)$ & $128(31.4)$ & 0.71 \\
\hline Ca channel blocker, $n(\%)$ & $62(11.5)$ & $44(9.8)$ & 0.39 & $72(20.3)$ & $53(13.0)$ & 0.007 \\
\hline Diuretics, $n(\%)$ & $259(47.9)$ & $129(28.6)$ & $<0.001$ & $156(43.9)$ & $151(37.0)$ & 0.051 \\
\hline Statins, $n(\%)$ & $16(3.0)$ & $45(10.0)$ & $<0.001$ & $81(22.8)$ & $94(23.0)$ & 0.94 \\
\hline Digoxin, $n(\%)$ & $70(12.9)$ & $16(3.5)$ & $<0.001$ & $45(12.7)$ & $15(3.7)$ & $<0.001$ \\
\hline Mean no. of cardiovascular medications (SD) & $1.5(1.3)$ & $1.1(1.2)$ & $<0.001$ & $1.9(1.5)$ & $1.5(1.4)$ & 0.001 \\
\hline Any antithrombotic agents, $n(\%)$ & $389(71.9)$ & $285(63.2)$ & 0.003 & $276(77.7)$ & $280(68.6)$ & 0.005 \\
\hline Warfarin, $n(\%)$ & $66(12.2)$ & $79(17.5)$ & 0.018 & $62(17.5)$ & $77(18.9)$ & 0.62 \\
\hline Dipyridamole, $n(\%)$ & $66(12.2)$ & $3(0.7)$ & $<0.001$ & $18(5.1)$ & $5(1.2)$ & 0.002 \\
\hline Dipyridamole/aspirin, $n(\%)$ & $40(7.4)$ & $26(5.8)$ & 0.31 & $33(9.3)$ & $41(10.0)$ & 0.73 \\
\hline Low-dose aspirin, $n(\%)$ & $317(58.6)$ & $176(39.0)$ & $<0.001$ & $176(49.6)$ & $156(38.2)$ & 0.002 \\
\hline Other agents, $n(\%)$ & $3(0.6)$ & $6(1.3)$ & 0.20 & $9(2.5)$ & $11(2.7)$ & 0.90 \\
\hline Mean no. of antithrombotic agents (SD) & $0.84(0.6)$ & $0.6(0.5)$ & $<0.001$ & $0.7(0.6)$ & $0.6(0.5)$ & $<0.001$ \\
\hline
\end{tabular}

Other agents included clopidogrel, enoxaparin, prasugrel and dalteparin

$A C E I$ angiotensin-converting enzyme inhibitor, $A R B$ angiotensin II receptor blocker, $M N A$ mini nutritional assessment, $S D$ standard deviation

and stroke, respectively. Residents with CHD or stroke and co-existing dementia had a lower prevalence in the use of any cardiovascular or antithrombotic agent, respectively. The decrease in cardiovascular medication use in residents with CHD in NHs over time appeared to be explained by the increasing proportion of residents with dementia for whom conservative prescribing is often advocated.

The prevalence of CHD declined overall in $\mathrm{NHs}$ and ALFs whereas the prevalence of stroke remained constant. In 2011, residents had significantly more disability, malnutrition and a higher prevalence of dementia compared with prior time points. This suggests that older people are entering NHs and ALFs frailer and more dependent in activities of daily living.
Long-term combination therapy for secondary prevention of CHD and risk factors is recommended irrespective of age by the AHA/ACCF and ESC guidelines $[16,18]$. The use of any cardiovascular agent significantly declined in both NHs and ALFs, including nitrates and diuretics that may increase the risk of falls [37]. Guideline-recommended therapies including $\beta$-blockers, ACEI/ARBs and low-dose aspirin remained $<50 \%$ in both settings in 2011 . This prevalence is lower than in recent studies in US and European NHs, which identified secondary preventative medications in approximately $63 \%$ and $77 \%$ of residents post-acute myocardial infarction and CHD, respectively $[3,26]$. The lower prevalence found in this study, particularly in the case of antihypertensive agents, reflects the complexity of prescribing in 

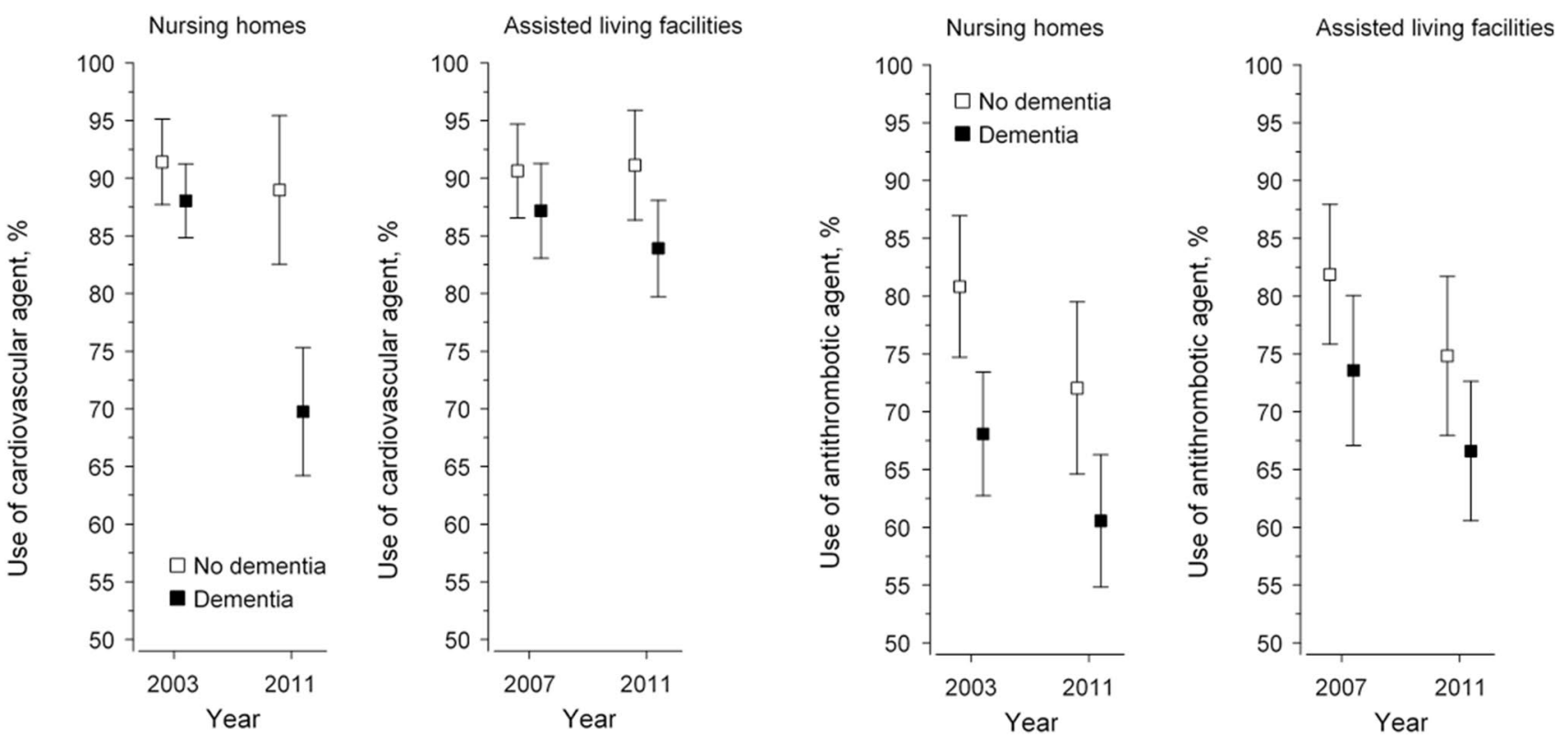

Fig. 1 Adjusted (gender, age, mobility, cancer and length of stay in the facility) use of cardiovascular and antithrombotic agents in residents with a history of coronary heart disease and stroke according to dementia, respectively

this population. Currently, the European Society of Hypertension provides guidance for systolic blood pressure (SBP) targets for older people based on evidence from the HYVET and SPRINT hypertension trials, which excluded LTCF residents [23]. It is unclear whether findings from these trials could be extrapolated to the LTCF population. The safety of combination antihypertensive therapy in LTCF residents has recently been questioned with findings from the PARTAGE study of 1127 LTCF residents aged over 80 years with low SBP $(<130 \mathrm{mmHg})$ identifying a higher risk of mortality with the use of two or more antihypertensive agents [22].

The use of any antithrombotic agent in this study significantly declined in both settings, however remained above $60 \%$. Although prescribing of antithrombotics for stroke prevention in older people $(\approx 70$ years and older $)$ is supported by the AHA and ASA [17], concerns over bleeding risk and under-representation of this population in clinical trials remains in clinical practice $[25,38,39]$. Warfarin, which may be used for the prevention of stroke in the setting of atrial fibrillation, increased significantly only in NHs. Its overall use was, however, relatively small, potentially due to concern over its monitoring requirements, risk of bleeding and concerns for falls [40]. Direct-acting oral anticoagulants were not identified in this study; however, future studies should investigate the impact of these medications on the prescribing of warfarin and other anticoagulants. Although statin use in NHs increased over time points, its use remains low in comparison with recent findings internationally in Australian [8, 41], Canadian [42] and European [3] NHs, which found a prevalence of up to $40 \%$. A review of randomized controlled trials (RCTs) and retrospective studies of statin use for secondary prevention in community-dwelling older people identified overall benefit in reduction of cardiovascular events and mortality in those aged $\geq 65$ to $<75$ years but minimal justification for its use in those aged $>85$ years [43]. Significant variability in the prescribing of statins has been identified, with studies in both Australian and Canadian NHs finding fewer statins prescribed for frail $\mathrm{NH}$ residents compared with non-frail residents [27, 42].

The proportion of residents with dementia increased over time in our study with $>60 \%$ of residents with CHD and stroke having a dementia diagnosis across settings in 2011. Among residents with CHD in NHs, the change in cardiovascular medication use over time was moderated by dementia, indicating conservative prescribing in this population. Although the severity of dementia for residents in this study was unclear, increased care requirements for residents residing in NHs compared with ALFs, which provide a more home-like environment, may indicate greater severity of dementia in this setting. Findings from this study are comparable to findings in the US community setting that found older adults with dementia were less likely to be prescribed secondary preventative medications for CHD than those without [44]. Prescribing of antithrombotics in NHs and ALFs was lower among residents with dementia than among those without dementia, indicating selective prescribing and deprescribing according to residents' clinical and frailty status. 
Increasing attention among clinicians on the rationalization of medications that may no longer be of benefit in residents with limited life expectancy and dementia has given rise to recommendations to improve appropriate prescribing in this population [14]. This includes advocacy for a palliative approach in residents with dementia with a focus on optimizing quality of life [14]. Consensus criteria by Holmes et al. has identified varying degrees of appropriateness of cardiovascular and antithrombotic agents in people with advanced dementia [14]. These included the use of $\beta$-blockers, calcium channel blockers, diuretics and ACEI/ARBs classified as sometimes appropriate; warfarin and antiarrhythmic agents including digoxin as rarely appropriate; and lipid-lowering medications and antiplatelet agents (excluding aspirin) as never appropriate [14]. Despite the availability of these consensus criteria, there is limited evidence from RCTs to inform appropriate medication use in this population. The apparent decline in adherence to guideline-recommended pharmacotherapy for the secondary prevention of CHD and stroke may represent good clinical practice and greater consideration for the ongoing risk and benefit of these medications in residents with limited life expectancy at high risk of adverse events.

There is currently minimal guidance for secondary prevention of CHD and stroke for frail older residents in LTCFs [24]. Instead, clinicians balance the risk of adverse events with the benefits in terms of preventing subsequent myocardial infarction and stroke and the impact on residents' quality of life. Although the AHA and ESC broadly support the use of secondary preventative medications in older people over 65 years with CHD and stroke [18, 24, 45, 46], there is an increasing view that their use may no longer be appropriate for residents with limited life expectancy and in LTCFs [28, 47]. Recently, the importance of research into prescribing patterns in vulnerable patient groups, such as those residing in NHs with dementia and limited life expectancy, has been emphasized [14, 48]. Increasing inclusion of these populations in studies examining the use of secondary preventative medications is required to inform evidencebased guidelines and recommendations in this population. Future studies should additionally explore the impact of the changes in prescribing of secondary preventative medications on outcomes including subsequent myocardial infarction, stroke and mortality.

\subsection{Strengths and Limitations}

This study utilized a large and representative sample size of residents from NHs and ALFs in Helsinki, Finland. The original sample comprised $>80 \%$ of all residents over the age of 65 years in NHs and over 60\% in ALFs [31]. Data were collected by trained nurses using the same protocol at all three time points. Medication use was defined as a point prevalence on the date of data collection using each resident's medication chart. This ensured we analysed actual medication use rather than prescribing or dispensing of medications. However, the use of point prevalence data limits our understanding of long-term medication usage over time and longitudinal data is required to explore this further. It is unclear when medications were commenced with respect to diagnoses. Cardiovascular medications may have also been prescribed for clinical indications other than CHD or stroke. The severity of dementia was not determined. This study did not investigate potential prescriber or facility-level factors that may have contributed to prescribing variability.

\section{Conclusion}

Declines in cardiovascular medication use in residents with CHD and dementia over time suggest Finnish physicians are adopting a more conservative approach to the management of cardiovascular disease in the $\mathrm{NH}$ population with dementia. Future research should explore the impact of these changes on outcomes in this population. There is a need for RCTs to further inform appropriate medication use in LTCFs.

\section{Compliance with Ethical Standards}

Funding $\mathrm{NJ}$ is supported through an Australian Government Research Training Program Scholarship. ECKT is supported by an NHMRCARC Dementia Research Development Fellowship (APP1107381). JSB is supported by an Australian National Health and Medical Research Council Dementia Leadership Fellowship.

Conflict of Interest Natali Jokanovic, Hannu Kautiainen, J. Simon Bell, Edwin C. K. Tan and Kaisu H. Pitkälä declare that they have no potential conflicts of interest that might be relevant to the contents of this manuscript.

Ethics Approval Ethics approval was obtained for all three studies by the Helsinki University Central Hospital Ethics Committee. The study was registered with the Monash University Human Research Ethics Committee. Written informed consent was obtained from residents and/ or their closest proxy to participate in each study.

\section{References}

1. American Heart Association and American Stroke Association. Heart disease and stroke statistics 2017-at a glance. 2017. https ://healthmetrics.heart.org/wp-content/uploads/2017/06/Heart -Disease-and-Stroke-Statistics-2017-ucm_491265.pdf. Accessed 21 Jan 2018

2. Cowman S, Royston M, Hickey A, Horgan F, McGee H, O'Neill D. Stroke and nursing home care: a national survey of nursing homes. BMC Geriatr. 2010;10:4.

3. Foebel AD, Liperoti R, Gambassi G, Gindin J, Ben Israel J, Bernabei R, et al. Prevalence and correlates of cardiovascular 
medication use among nursing home residents with ischemic heart disease: results from the SHELTER study. J Am Med Dir Assoc. 2014;15(6):410-5.

4. Malek Makan A, van Hout H, Onder G, Finne-Soveri H, van der Roest H, van Marum R. Prevalence of preventive cardiovascular medication use in nursing home residents. Room for deprescribing? The SHELTER study. J Am Med Dir Assoc. 2017;18(12):1037-42.

5. Australian Institute of Health and Welfare. Australia's health 2016. 2016. https://www.aihw.gov.au/getmedia/9844cefb-7745-4dd89ee2-f4d1c3d6a727/19787-AH16.pdf.aspx ?inline=true. Accessed 21 Jan 2018.

6. Benjamin EJ, Blaha MJ, Chiuve SE, Cushman M, Das SR, Deo R, et al. Heart disease and stroke statistics-2017 update: a report from the American Heart Association. Circulation. 2017;135(10):e146-603.

7. Jokanovic N, Tan EC, Dooley MJ, Kirkpatrick CM, Bell JS. Prevalence and factors associated with polypharmacy in longterm care facilities: a systematic review. J Am Med Dir Assoc. 2015;16(6):535.e1-12.

8. Jokanovic N, Jamsen KM, Tan ECK, Dooley MJ, Kirkpatrick CM, Bell JS. Prevalence and variability in medications contributing to polypharmacy in long-term care facilities. Drugs Real World Outcomes. 2017;4(4):235-45.

9. Liu E, Dyer SM, O’Donnell LK, Milte R, Bradley C, Harrison $\mathrm{SL}$, et al. Association of cardiovascular system medications with cognitive function and dementia in older adults living in nursing homes in Australia. J Geriatr Cardiol. 2017;14(6):407-15.

10. Johnell K, Fastbom J. Comparison of prescription drug use between community-dwelling and institutionalized elderly in Sweden. Drugs Aging. 2012;29(9):751-8.

11. Shah SM, Carey IM, Harris T, Dewilde S, Cook DG. Quality of chronic disease care for older people in care homes and the community in a primary care pay for performance system: retrospective study. BMJ. 2011;342:d912.

12. Centre for Disease Control and Prevention. Long-term care providers and services users in the United States: data from the national study of long-term care providers, 2013-2014. 2016. https://www.cdc.gov/nchs/data/series/sr_03/sr03_038.pdf. Accessed 21 Jan 2018.

13. Onder G, Carpenter I, Finne-Soveri H, Gindin J, Frijters D, Henrard JC, et al. Assessment of nursing home residents in Europe: the services and health for elderly in long TERm care (SHELTER) study. BMC Health Serv Res. 2012;12:5.

14. Holmes HM, Sachs GA, Shega JW, Hougham GW, Cox Hayley D, Dale W. Integrating palliative medicine into the care of persons with advanced dementia: identifying appropriate medication use. J Am Geriatr Soc. 2008;56(7):1306-11.

15. Tan EC, Bell JS, Lu CY, Toh S. National trends in outpatient antihypertensive prescribing in people with dementia in the United States. J Alzheimers Dis. 2016;54(4):1425-35.

16. Smith SC Jr, Benjamin EJ, Bonow RO, Braun LT, Creager MA, Franklin BA, et al. AHA/ACCF secondary prevention and risk reduction therapy for patients with coronary and other atherosclerotic vascular disease: 2011 update: a guideline from the American Heart Association and American College of Cardiology Foundation endorsed by the World Heart Federation and the Preventive Cardiovascular Nurses Association. J Am Coll Cardiol. 2011;58(23):2432-46.

17. Kernan WN, Ovbiagele B, Black HR, Bravata DM, Chimowitz MI, Ezekowitz MD, et al. Guidelines for the prevention of stroke in patients with stroke and transient ischemic attack: a guideline for healthcare professionals from the American Heart Association/ American Stroke Association. Stroke. 2014;45(7):2160-236.

18. Montalescot G, Sechtem U, Achenbach S, Andreotti F, Arden C, Budaj A, et al. 2013 ESC guidelines on the management of stable coronary artery disease: the Task Force on the management of stable coronary artery disease of the European Society of Cardiology. Eur Heart J. 2013;34(38):2949-3003.

19. Ferri C, Ferri L, Desideri G. Management of hypertension in the elderly and frail elderly. High Blood Press Cardiovasc Prev. 2017;24(1):1-11.

20. Beckett NS, Peters R, Fletcher AE, Staessen JA, Liu L, Dumitrascu D, et al. Treatment of hypertension in patients 80 years of age or older. N Engl J Med. 2008;358(18):1887-98.

21. Williamson JD, Supiano MA, Applegate WB, Berlowitz DR, Campbell RC, Chertow GM, et al. Intensive vs standard blood pressure control and cardiovascular disease outcomes in adults aged $\geq 75$ years: a randomized clinical trial. JAMA. 2016;315(24):2673-82

22. Benetos A, Labat C, Rossignol P, Fay R, Rolland Y, Valbusa $\mathrm{F}$, et al. Treatment with multiple blood pressure medications, achieved blood pressure, and mortality in older nursing home residents: the PARTAGE study. JAMA Intern Med. 2015;175(6):989-95.

23. Kjeldsen SE, Stenehjem A, Os I, Van de Borne P, Burnier M, Narkiewicz K, et al. Treatment of high blood pressure in elderly and octogenarians: european Society of Hypertension statement on blood pressure targets. Blood Press. 2016;25(6):333-6.

24. Fleg JL, Forman DE, Berra K, Bittner V, Blumenthal JA, Chen $\mathrm{MA}$, et al. Secondary prevention of atherosclerotic cardiovascular disease in older adults: a scientific statement from the American Heart Association. Circulation. 2013;128(22):2422-46.

25. Bushnell CD, Colon-Emeric CS. Secondary stroke prevention strategies for the oldest patients: possibilities and challenges. Drugs Aging. 2009;26(3):209-30.

26. Zullo AR, Sharmin S, Lee Y, Daiello LA, Shah NR, John Boscardin $\mathrm{W}$, et al. Secondary prevention medication use after myocardial infarction in U.S. nursing home residents. J Am Geriatr Soc. 2017;65(11):2397-404.

27. Kantoch A, Gryglewska B, Wojkowska-Mach J, Heczko P, Grodzicki T. Treatment of cardiovascular diseases among elderly residents of long-term care facilities. J Am Med Dir Assoc. 2018;19:428-32.

28. Poudel A, Yates P, Rowett D, Nissen LM. Use of preventive medication in patients with limited life expectancy: a systematic review. J Pain Symptom Manage. 2017;53(6):1097-110.e1.

29. Hosia-Randell H, Pitkala K. Use of psychotropic drugs in elderly nursing home residents with and without dementia in Helsinki, Finland. Drugs Aging. 2005;22(9):793-800.

30. Muurinen S, Savikko N, Soini H, Suominen M, Pitkala K. Nutrition and psychological well-being among long-term care residents with dementia. J Nutr Health Aging. 2015;19(2):178-82.

31. Pitkala KH, Juola AL, Hosia H, Teramura-Gronblad M, Soini H, Savikko N, et al. Eight-year trends in the use of opioids, other analgesics, and psychotropic medications among institutionalized older people in Finland. J Am Med Dir Assoc. 2015;16(11):973-8.

32. Suominen MH, Sandelin E, Soini H, Pitkala KH. How well do nurses recognize malnutrition in elderly patients? Eur J Clin Nutr. 2009;63(2):292-6.

33. Sanford AM, Orrell M, Tolson D, Abbatecola AM, Arai H, Bauer $\mathrm{JM}$, et al. An international definition for "nursing home". J Am Med Dir Assoc. 2015;16(3):181-4.

34. WHO Collaborating Centre for Drug Statistics Methodology. Anatomical Therapeutic Chemical (ATC) Classification Index. 2016. https://www.whocc.no/atc_ddd_index/. Accessed 21 Jan 2018.

35. Charlson ME, Pompei P, Ales KL, MacKenzie CR. A new method of classifying prognostic comorbidity in longitudinal studies: development and validation. J Chronic Dis. 1987;40(5):373-83.

36. Guigoz Y, Lauque S, Vellas BJ. Identifying the elderly at risk for malnutrition. The Mini Nutritional Assessment. Clin Geriatr Med. 2002;18(4):737-57. 
37. Berry SD, Mittleman MA, Zhang Y, Solomon DH, Lipsitz LA, Mostofsky E, et al. New loop diuretic prescriptions may be an acute risk factor for falls in the nursing home. Pharmacoepidemiol Drug Saf. 2012;21(5):560-3.

38. Tay KH, Lane DA, Lip GY. Challenges facing anticoagulation among the elderly and frail. Age Ageing. 2009;38(2):140-2.

39. Lindley RI. Stroke prevention in the very elderly. Stroke. 2018;49(3):796-802.

40. Neidecker M, Patel AA, Nelson WW, Reardon G. Use of warfarin in long-term care: a systematic review. BMC Geriatr. 2012;12:14.

41. Korhonen MJ, Ilomaki J, Sluggett JK, Brookhart MA, Visvanathan R, Cooper T, et al. Selective prescribing of statins and the risk of mortality, hospitalizations, and falls in aged care services. J Clin Lipidol. 2018;12(3):652-61.

42. Campitelli MA, Maxwell CJ, Giannakeas V, Bell CM, Daneman $\mathrm{N}$, Jeffs $\mathrm{L}$, et al. The variation of statin use among nursing home residents and physicians: a cross-sectional analysis. J Am Geriatr Soc. 2017;65(9):2044-51.

43. Ruscica M, Macchi C, Pavanello C, Corsini A, Sahebkar A, Sirtori CR. Appropriateness of statin prescription in the elderly. Eur J Intern Med. 2018;50:33-40.
44. Fowler NR, Barnato AE, Degenholtz HB, Curcio AM, Becker JT, Kuller LH, et al. Effect of dementia on the use of drugs for secondary prevention of ischemic heart disease. J Aging Res. 2014;2014:897671.

45. Alhusban A, Fagan SC. Secondary prevention of stroke in the elderly: a review of the evidence. Am J Geriatr Pharmacother. 2011;9(3):143-52.

46. Kirchhof P, Benussi S, Kotecha D, Ahlsson A, Atar D, Casadei B, et al. 2016 ESC Guidelines for the management of atrial fibrillation developed in collaboration with EACTS. Eur J Cardiothorac Surg. 2016;50(5):e1-88.

47. Maddison AR, Fisher J, Johnston G. Preventive medication use among persons with limited life expectancy. Prog Palliat Care. 2011;19(1):15-21.

48. Tan ECK, Sluggett JK, Johnell K, Onder G, Elseviers M, Morin $\mathrm{L}$, et al. Research priorities for optimizing geriatric pharmacotherapy: an international consensus. J Am Med Dir Assoc. 2018;19(3):193-9.

\section{Affiliations}

\section{Natali Jokanovic ${ }^{1,2} \cdot$ Hannu Kautiainen ${ }^{3} \cdot$ J. Simon Bell ${ }^{1} \cdot$ Edwin C. K. Tan $^{1,4,5} \cdot$ Kaisu H. Pitkälä $^{3}$}

1 Centre for Medicine Use and Safety, Faculty of Pharmacy and Pharmaceutical Sciences, Monash University, Melbourne, Australia

2 Pharmacy Department, Alfred Hospital, Melbourne, Australia

3 Department of General Practice and Unit of Primary Health Care, University of Helsinki and Helsinki University Central Hospital, Helsinki, Finland
4 School of Pharmacy, Faculty of Medicine and Health, The University of Sydney, Sydney, Australia

5 Aging Research Centre, Department of Neurobiology, Care Sciences and Society, Karolinska Institute and Stockholm University, Stockholm, Sweden 